\title{
Emergy-based complexity measures in natural and social systems
}

\author{
S. Ulgiati ${ }^{a}, *$ M. Ascione ${ }^{a}$, A. Zucaro ${ }^{a}$, L. Campanella $^{b}$ \\ a Department of Sciences for the Environment, "Parthenope" University of Napoli, Italy \\ b Department of Chemistry, University of Roma 1 "La Sapienza", Roma, Italy
}

\section{A R T I C L E I N F O}

\section{Article history:}

Received 2 July 2010

Received in revised form

29 December 2010

Accepted 30 December 2010

\section{Keywords:}

Emergy

Complexity

Diversity

Urban systems

\begin{abstract}
A B S T R A C T
A complexity indicator based on the diversity of energy and resource uses by a system is proposed in this paper. The indicator is an emergy-based index of complexity derived from a modified Shannon information formula that provides a quantitative assessment of the diversity of sources. The emergy approach assigns to each driving input a weight that derives from the environmental work performed by nature in order to generate such resource. This quality assessment goes far beyond the simple accounting of mass and energy of input flows, but takes into proper account their interlinkage with the biosphere dynamics. The rationale of the proposed indicator is that complexity cannot be assessed by simply counting individuals, species and processes, but requires that focus is placed on several aspects of resource flows, namely their amount, frequency, and quality. Different mixes of emergy input flows originate different levels of growth and complexity. Systems that only rely on a small set of sources out of the large number potentially available possess a built-in fragility, that may determine their collapse in times of shrinking or changing resource basis. For validation purpose, the proposed indicator was applied to the performance of selected national economies (Nicaragua, Latvia, Denmark and Italy) in selected years and of the urban system of Roma (Italy) over a forty-year (1962-2002) historical series. Results point out an increasing complexity of the urban system of Rome over time, while a lower complexity was calculated for the investigated national systems as a whole (likely effect of nationwide averaging), with Italy ranking highest and Latvia lowest. The same assessment performed for the Italian agricultural system over a twenty-year time series (1985-2006) shows a decline of the emergy-adjusted Shannon indicator from about $75 \%$ down to $62 \%$, while the decline was from $73 \%$ to $63 \%$ for the agriculture of Campania region (southern Italy).
\end{abstract}

(c) 2011 Elsevier Ltd. All rights reserved.

\section{Introduction: state-of-the-art and innovative measures of diversity}

Complexity of systems has long been represented by "information-theory" measures. For example, complexity in bits is the number of yes-no decisions required to define a configuration and is expressed on a logarithmic scale. In short, the informationtheory measures the logarithm of the possibilities among the parts and connections. For example, there is great complexity at the small-scales of molecules and heat, where information-theory measure on a logarithmic scale is molecular entropy. However, information theory measures do not differentiate between large scale complexity that operates a macroscopic natural or social system and small scale complexity with the same number of parts that have smaller influence on the global dynamics of the larger system in which they are embedded. Information-theory measures do not distinguish this complexity on small molecular

\footnotetext{
* Corresponding author. Tel.: +390577 234232; fax: +390577 234254.

E-mail address: sergio.ulgiati@uniparthenope.it (S. Ulgiati).
}

scale from that found on a large ecological scale. The awareness of such a limit gave rise to innovative measures, all based on Shannon information formula and all trying to overcome the scale problem by means of concepts that incorporate time and spatial scales into the assessment. Two of such different measures are reviewed in the following.

\subsection{Diversity in ecosystems}

While studying the interactions between components of ecosystems, Margalef (1968) suggested that "a measure of the aggregation of the ecosystems may be found in the average distance between the place of energy input and the energy sink. The distance can probably be measured either in terms of space or of time". He also pointed out that "there is some energy exchange between...subsystems in the sense that the less-organized subsystem gives energy to the more-organized, and, in the process of exchange, some information in the less-organized is destroyed and some information is gained by the already more-organized". The transfer of energy between subsystems originates a hierarchical organization of components, where many components at the lower 
levels 'pay the bill' of supporting few components at the higher ones. Finally, Margalef compared an ecosystem to a message transmitted by means of a certain code. Borrowing expressions from information theory, he defined the average information content per individual as

$D=-\sum p_{i} \log _{2} p_{i}=\frac{1}{N} \log _{2}\left[\frac{N !}{N_{a} ! N_{b} !, \ldots, N_{s} !}\right]$

where $N_{a}, N_{b}, \ldots, N_{s}$ are number of individuals of species $a, b, \ldots$, $s ; N$ is the total number of individuals; $p_{i}$ is the probability that one individual belongs to species $i$. The minimum of these expressions is when all individuals belong to the same species, while the maximum is when each individual belongs to a different species. These cases are both improbable in nature, while actual values are in between: Margalef called this measure "diversity" and discussed it as a measure of organization, correlating it with the size of the sample, the energy flow per unit biomass and the entropy production in sustaining a unit of biomass in the ecosystem. Changes in diversity have been discussed by Margalef as useful and clear indicators of movement of the ecosystem back and forth on the succession pathway.

\subsection{Thermodynamic depth}

Lloyd and Pagels (1988) developed a measure of complexity named thermodynamic depth, based on a probabilistic metric in information theory. The author's concern was to avoid a definition of complexity based on additive properties of the individual objects. If so, complexity could be increased just by increasing the number of the objects, thus making complexity to proliferate very cheaply. Rather, "complexity must be a function of the process. . .that brought the object into existence. . .Seven bulls need not be too much more complex than one bull. It took billion of years for the earth to evolve one bull; but one bull and a few compliant cows will produce seven bulls relatively speedily" (Lloyd and Pagels, op. cit.). Given a macroscopic state, $d$, of a system $D$ that can be reached through $n$ different trajectories, experiments can assign a probability $p_{i}$ to the $i$-th trajectory. A trajectory of macroscopic states of a system $D$ is defined as an ordered set of macroscopic states $a_{i}, b_{j}, \ldots, c_{k}$, such that $D$ has been measured to be in the state $a_{i}$ at time $t_{1}$, in the state $b_{j}$ at time $t_{2} \geq t_{1}, \ldots$, in the state $c_{k}$ at time $t_{n} \geq t_{n-1}$. Lloyd and Pagels proved that the complexity of the state $d$, that has been reached through the $i$-th possible trajectory, is measured by the function

$D(d)=-k \ln P_{i}$

and that the average complexity of a state is proportional to the Shannon entropy of the set of trajectories that can lead to that state,

$S=-k \sum p_{i} \ln p_{i}$

where $k$ is an arbitrary constant. They named depth the function $D(d)$ and defined complexity as the pathway from the reference state to the present one. Considering all the possible trajectories that may lead to the present system's state, they calculate the socalled thermodynamic depth $D_{T}$ of a state, as the amount of entropy that the system has pumped from the 'relevant' degrees of freedom (those that needed to be constrained for the system to evolve into the state $d$ ) to 'irrelevant' degrees of freedom (the remaining ones) in the course of constructing the state $d$. The thermodynamic depth can be shown to be "proportional to the amount of information needed to identify the trajectory that leads to $d$ given the information that the system is in $d$ already" (Lloyd and Pagels, op. cit.):

$D_{T}(d)=\left(k_{B} \ln 2\right)\left[I_{o}(d)-I(d)\right]$
In other words, the depth as a measure of system's complexity is greater when the trajectory leading to the state $d$ is unlikely or when the number of discarded trajectories is higher.

\section{Incorporating quality and hierarchies within complexity measures}

The diversity and thermodynamic depth indices developed by Margalef and by Lloyd and Pagels address the important issue of complexity from an information theory and probabilistic point of view, by looking at the organization and the development trajectories of a system's state, which is already a major step ahead in complexity assessment. Brown et al. (2006) and Brown and Cohen (2007), building on Odum's emergy synthesis approach (Odum, 1996) identified a main problem in the lack for an overall measure of biodiversity at various levels of an ecological hierarchy, due to the fact that Shannon-like diversity measures cannot be summed. If they were summed - these authors point out - bacteria and other small animals and plants would dominate the resulting diversity to the total neglect of the larger species. What is needed is to develop a quantitative evaluation of total biodiversity within regions or ecosystems by weighting biodiversity at each hierarchical level by means of trophic-level quality indicators (identified as "transformities") derived from the emergy approach. In this way measures of biodiversity can be quantitatively compared and changes resulting from species loss can be scaled based on transformities. A more realistic picture of total biodiversity emerges and allows comparison of losses and gains that result from changes in ecological health.

\subsection{Emergy and transformity: concepts and definitions}

Odum (1996) introduced the concept of emergy in order to account for the quality of incoming energy and resources, i.e., for the environmental services supporting a process as well as for their convergence through a chain of energy and matter transformations in both space and time. By definition, emergy is the amount of energy of one type (usually solar) that is directly or indirectly required to provide a given flow or storage of energy or matter. Solar emergy is expressed in solar equivalent joules (seJ, or solar emjoules). The solar emergy required to generate a unit flow or a storage of available energy is called solar transformity and is expressed as solar emergy joules per joule of output flow or product $(\mathrm{se} / \mathrm{J})$. The transformity of solar radiation is assumed equal to one by definition $(1.0 \mathrm{seJ} / \mathrm{J})$, while the transformities of all the other flows and storages (including those related to human societies) are calculated based on their convergence patterns through the biosphere hierarchy.

While it is true that all energy can be converted to heat, it is not true that one form of energy is substitutable for another in all situations. For instance, plants cannot substitute fossil fuel for sunlight in photosynthetic production, nor can humans substitute sunlight energy for food or water. It should be obvious that a characteristics that makes an energy flow usable by one set of transformation processes makes it unusable for another set. Thus, quality is related to a form of energy and to its concentration; where higher quality is somewhat synonymous with higher concentration of energy and results in greater flexibility. So, wood is more concentrated than detritus, coal more concentrated than wood, and electricity more concentrated than coal. As a consequence, the quality (concentration, wave-length, pulsing, etc.) of incoming energy makes it able to drive different forms of complexity in recipient systems. The emergy approach makes the quality assessment possible and provides concepts and quantitative calculation procedures rooted in systems ecology and irreversible thermodynamics. 
Table 1

Calculation procedure of the emergy-based Source Diversity (SD) index for the city of Rome (year 2002).

\begin{tabular}{|c|c|c|c|c|}
\hline Emergy flow, $U_{i}$ & Amount & $U_{i} / U$ & $\log \left(U_{i} / U\right)$ & $S D_{i}$ \\
\hline Renewable emergies (sun, wind, rain) & $3.91 \mathrm{E}+20$ & 0.00 & -2.55 & 0.01 \\
\hline Top soil (erosion, weathering) & $1.76 \mathrm{E}+21$ & 0.01 & -1.90 & 0.02 \\
\hline Gasoline & $6.52 \mathrm{E}+21$ & 0.05 & -1.33 & 0.06 \\
\hline Diesel fuel & $7.80 \mathrm{E}+21$ & 0.06 & -1.25 & 0.07 \\
\hline LPG (Liquid petroleum gas) & $1.05 \mathrm{E}+21$ & 0.01 & -2.12 & 0.02 \\
\hline Heavy oil for domestic heating & $6.02 \mathrm{E}+20$ & 0.00 & -2.36 & 0.01 \\
\hline Natural gas for cooking & $3.49 \mathrm{E}+20$ & 0.00 & -2.60 & 0.01 \\
\hline Natural gas for heating & $1.42 \mathrm{E}+21$ & 0.01 & -1.99 & 0.02 \\
\hline Natural gas, other uses & $2.11 \mathrm{E}+21$ & 0.02 & -1.82 & 0.03 \\
\hline Electricity & $9.05 E+21$ & 0.07 & -1.18 & 0.08 \\
\hline Water (from aqueduct) & $1.23 \mathrm{E}+21$ & 0.01 & -2.05 & 0.02 \\
\hline Fish & $4.99 \mathrm{E}+21$ & 0.04 & -1.44 & 0.05 \\
\hline Meat & $2.05 E+21$ & 0.01 & -1.83 & 0.03 \\
\hline Fruits and vegetables & $2.45 \mathrm{E}+20$ & 0.00 & -2.75 & 0.00 \\
\hline Milk, cheese and other derivatives & $2.15 E+21$ & 0.02 & -1.81 & 0.03 \\
\hline Cereals and derivatives & $8.03 E+19$ & 0.00 & -3.24 & 0.00 \\
\hline Wine and alcoholics & $5.38 \mathrm{E}+19$ & 0.00 & -3.41 & 0.00 \\
\hline Olive and seed oils & $8.26 \mathrm{E}+21$ & 0.06 & -1.22 & 0.07 \\
\hline Steel and iron & $7.82 \mathrm{E}+21$ & 0.06 & -1.25 & 0.07 \\
\hline Copper & $1.67 \mathrm{E}+20$ & 0.00 & -2.92 & 0.00 \\
\hline Aluminum & $5.21 \mathrm{E}+19$ & 0.00 & -3.42 & 0.00 \\
\hline Cement (Portland) & $3.41 \mathrm{E}+21$ & 0.02 & -1.61 & 0.04 \\
\hline Rocks and sediments, building sector & $2.68 \mathrm{E}+22$ & 0.19 & -0.71 & 0.14 \\
\hline Glass & $8.23 E+20$ & 0.01 & -2.23 & 0.01 \\
\hline Plastics & $5.42 \mathrm{E}+21$ & 0.04 & -1.41 & 0.06 \\
\hline Asphalt & $1.40 \mathrm{E}+21$ & 0.01 & -2.00 & 0.02 \\
\hline Chemicals & $9.68 \mathrm{E}+20$ & 0.01 & -2.16 & 0.02 \\
\hline Wood & $4.04 \mathrm{E}+20$ & 0.00 & -2.54 & 0.01 \\
\hline Textiles & $6.87 \mathrm{E}+21$ & 0.05 & -1.30 & 0.06 \\
\hline Paper and derivatives & $3.20 \mathrm{E}+21$ & 0.02 & -1.64 & 0.04 \\
\hline Fertilizers & $2.23 \mathrm{E}+21$ & 0.02 & -1.79 & 0.03 \\
\hline Services for imports & $1.96 \mathrm{E}+22$ & 0.14 & -0.85 & 0.12 \\
\hline Imported labor (Commuters) & $9.15 \mathrm{E}+21$ & 0.07 & -1.18 & 0.08 \\
\hline Total emergy used annually, $U$ & $1.38 \mathrm{E}+23$ & & & \\
\hline Quality-adjusted Shannon indicator of source diversity, $S D$ & & 1.22 & & \\
\hline
\end{tabular}

\subsection{A system-level diversity index}

Brown et al. (2006) pointed out two conceptual problems with the standard use of the Shannon equation in ecology: "first, it uses stocks instead of flows, and second one cannot compute whole system diversity since it ignores the hierarchical organization of ecosystems (the equation is maximized when the probability of observing each component is equal)". They suggested a quality-adjusted Shannon diversity, where the probability of each individual or species is replaced by an emergy importance value (EIV = emergy of component/emergy of all components):

$E I V_{i}=\frac{N P_{i} * \tau_{i}}{\sum_{i} N P_{i} * \tau_{i}}$

where $E I V_{i}$ is the emergy importance value, $N P_{i}$ the net production $(\mathrm{J} / \mathrm{yr})$ and $\tau_{i}(\mathrm{se} / \mathrm{J})$ is the computed transformity of the $i$-th component. The importance value is the relative contribution of each component to the total emergy flow through all biotic components. By replacing the probability $p_{i}$ with the $E I V_{i}$ the Shannon information index translates into an ecosystem-scale Shannon diversity index as follows:

Biodiversity $=-\sum_{i=1}^{n} E I V_{i} * \log \left[E I V_{i}\right]$

Such a biodiversity index is maximized when the emergy on each pathway, and therefore each component's $E I V_{i}$, is equal, as postulated by Odum (1996), i.e., emergy flow evenness across all components being the condition for all network systems to maximize power. By applying such an evaluation method, Brown et al. (2006) calculated an operating efficiency at $42 \%$ of maximum potential diversity for the Florida Everglades ecosystem. Finally, it should be noted that, by using the emergy approach, a built-in memory of all trial-and-error steps of the investigated system is included in the assessment, in so taking into proper account the environmental cost of the trajectory followed by the system up to the present state.

\section{Diversity of input flows as complexity driver}

A histogram showing all emergy inputs hierarchically ordered by quality by means of their transformity values is named an emergy signature. A signature is a picture of the driving forces, which support a system development. Changes in ecosystem complexity can result from alterations in driving energy signature, or inflows of a high quality stressor such as pollutants, or unsustainable activity like over-harvesting. In each case, there is a consequent change in the pattern of energy flows supporting organization.

Ulgiati and Brown $(2006,2009)$ investigated the relation between emergy signatures (diversity of sources) and the development state of human-dominated systems. It is apparent that the presence or absence of a given input flow typology such as electricity heavily affects a system's performance (e.g., the possibility of industrial development, computer use and TV broadcasting). Fully natural systems or developing economies are in general driven by a set of flows mainly characterized by low transformities (e.g., a mangrove ecosystem or the economic systems of less industrialized countries), while highly industrialized systems and economies rely on high-transformity flows (a hectare of intensive corn agriculture or the economy of a very developed country). The signatures of such systems are very unbalanced, since they most often rely on a small number of flows, that are location or technology specific and the availability of which might not be guar- 
anteed on a long time scale, due to changes in economic or climatic conditions.

Ulgiati and Brown $(2006,2009)$ compared the signatures of a hectare of mangrove ecosystem and a hectare of intensive corn in Florida, as well as the signatures of the economic systems of Nicaragua, Latvia, Denmark and Italy. In all cases, very diverse categories of driving forces were detected. The signature of the mangrove ecosystem showed only environmental driving forces, while the industrialized corn was mostly driven by fossil fuels, goods, labor and services. The findings of the study suggested that an ecosystem development is not only driven and determined by the total emergy inflow, but also by the quality of the different inflows. In the first case, the ecosystem is driven towards ecological biodiversity and complexity, with an average transformity of $2.47 \mathrm{E}+04 \mathrm{seJ} / \mathrm{J}$; in the second case, the system is pushed towards a state with lower diversity and higher net productivity per hectare. In spite of such a higher productivity, the transformity of the industrialized corn was $1.26 \mathrm{E}+05 \mathrm{se} / \mathrm{J}$, indicating a much lower conversion efficiency. Similarly, in the comparison between Nicaraguan, Latvia, Danish and Italian economies the emergy signatures were very different. The Nicaraguan and Latvian signatures indicated rural countries, mainly supported by fully environmental emergy flows, while instead the opposite was true for Denmark and Italy. On the one hand, we have two nations still rich with environmental complexity, while on the other hand we have two nations characterized by higher technological complexity. Until the present state is stable (e.g., no increase of oil scarcity) the structures of these societies remain unchanged and stable. If the availability of some of these input flows starts declining, societies may find it difficult to replace them and face stability and resiliency problems.

\subsection{An emergy-based index of source diversity}

What is still missing in the evaluation of source diversity is the possibility to generate a quantitative index that can be used for comparison of systems and for the assessment of a system change over time. A suitable source diversity index can be developed by adapting the Shannon information formula to the emergy inflows, similar to the quality-adjusted Shannon biodiversity index developed by Brown et al. (2006). Same Eqs. (5) and (6) can be used, of course based on a slightly different definition of EIVS ${ }_{i}$ (emergy importance value of sources):

$E I V S_{i}-\left(\frac{U_{i}}{\sum_{i} U_{i}}\right)$

where $U_{i}=$ emergy used $=($ amount of $i$-th flow; $\mathrm{J}$ or $\mathrm{g}) \times$ (emergy intensity of the $i$-th flow; seJ/J or seJ/g). By replacing in Eq. (6) the $E I V S_{i}$ defined in Eq. (7) a measure of diversity of sources is obtained:

source diversity $(S D)=-\sum_{i}\left(E I V S_{i} * \log E I V S_{i}\right)$

The value of $S D$ is maximum when all input flows have the same $E I V S_{i}$, as with the biodiversity index in Eq. (6). The number of input flows included in the calculation procedure affects the value of each $E I V S_{i}$ (since it must be $\sum_{i} E I V S_{i}=1$ ) thus also affecting the maximum value of the source diversity index, $S D_{\max }$. Depending on how input flows are aggregated, $S D_{\max }$ may be different for the same system, but the time evolution of the system's $S D$ is not affected. In fact, if aggregation choices do not change, $S D_{\max }$ also remains constant, and the calculated $S D$ variations reflect the actual changes of the driving flows over time. For the sake of simplicity, we can define an aggregated index, namely a "diversity ratio" defined as $\triangle S D$ $(\%)=100 *\left(S D / S D_{\max }\right)$. The conditions that determine $S D_{\max }$ (emergy flow evenness) do not mean that the system receives the same amount of each input resource. Depending on the transformities of

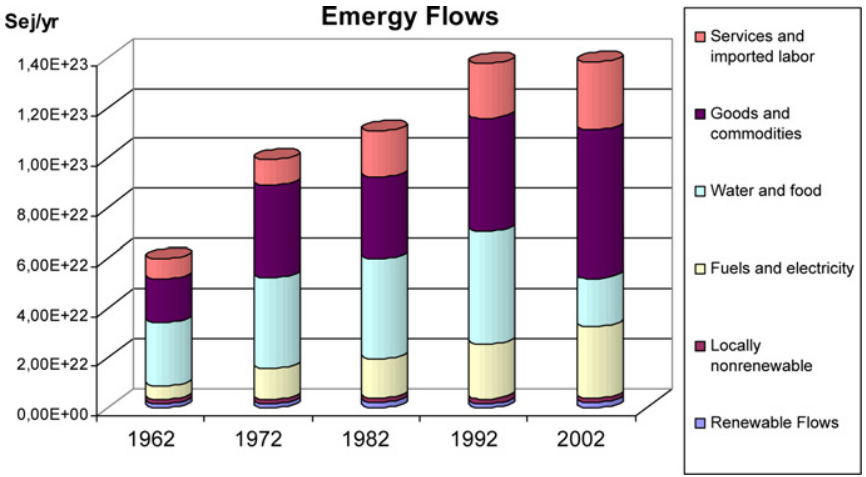

Fig. 1. Emergy support to the urban system of Rome (Italy) from 1962 to 2002.

each flow, the requisite of emergy evenness translates into an adequate matching of large amounts of flows with low transformity and small amounts of flows with high transformity, for maximum power output. As a consequence, a $S D$ closer to $S D_{\max }$ (and therefore a ratio $S D / S D_{\max }$ closer to 1 ) suggests higher system resilience.

\section{Results: source diversity in urban, agricultural and economic systems}

In order to test the potential of the source diversity index, we first applied the suggested calculation procedure to the emergy inflows driving the development of the urban system of Rome over the period 1962-2002 (Ascione et al., 2009). Fig. 1 shows the increase of the urban emergy use over time. Apparently, a plateau was reached in the last decade (1992-2002) investigated. It can be seen that although the total emergy use was constant in such period, the breakdown of the different driving forces changed significantly, with an increase of the imported goods, energy and services, and a sharp decrease of water and food use. It should be noticed that a variation of the emergy of a given typology (e.g., food) may derive from a change in the mix of components (i.e., more vegetarian instead of carnivore diet), characterized by different transformities and therefore translating into a change of the related emergy value. The value of $S D_{\max }$ is affected by the number of categories considered in each study as well as by their aggregation, that translate into different EIVS $S_{i}$ in Eqs. (7) and (8). Such categories are not necessarily the same for each system, due to the fact that the driving forces are location and technology specific or may have been differently aggregated by the analyst. As a consequence, the absolute values $S D$ and $S D_{\max }$ are not very telling, while what matters is their ratio $\triangle S D(\%)$ that provides a measure of the distance between the present state and the optimum state characterized by the matching of low and high quality sources.

Fig. 2 shows the emergy signature of Rome in the year 2002, that suggests a large reliance on developed and technological sources of emergy. The application of Eq. (8) to data from Ascione et al. (2009) is shown in Table 1 for the year 2002 and translates into the source diversity indices of Rome (years 1962-2002) shown in Table 2. It appears that the ratio $\triangle S D(\%)$ increases from about $75.6 \%$ in the year 1962 to $80.3 \%$ in the year 2002, suggesting an

Table 2

Trend of Shannon quality-adjusted source diversity indices of the urban system of Rome (years 1962-2002).

\begin{tabular}{lrrrrr}
\hline & 1962 & 1972 & 1982 & 1992 & 2002 \\
\hline$S D$ & 1.15 & 1.12 & 1.20 & 1.15 & 1.22 \\
$S D_{\max }$ & 1.52 & 1.52 & 1.52 & 1.52 & 1.52 \\
$S D / S D_{\max }(\%)$ & $75.7 \%$ & $73.7 \%$ & $78.9 \%$ & $75.7 \%$ & $80.3 \%$ \\
\hline
\end{tabular}




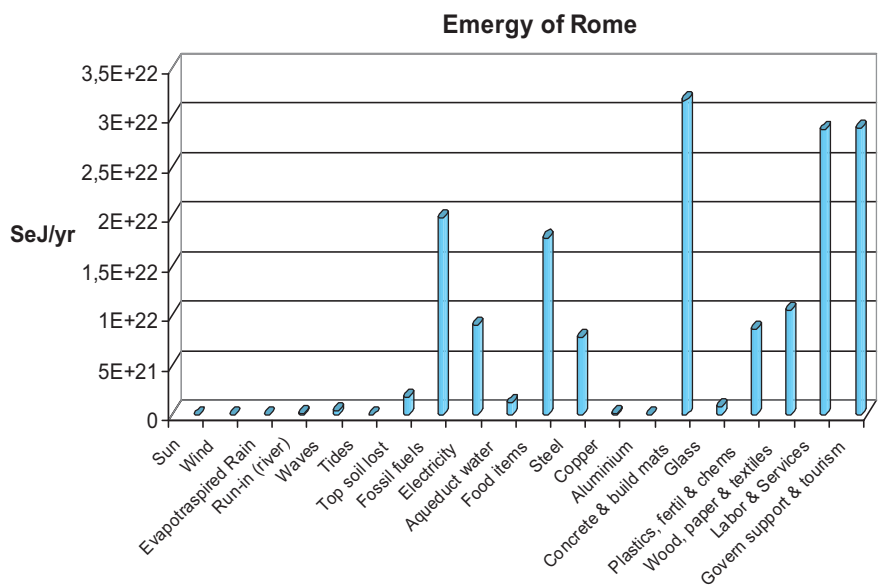

Fig. 2. Emergy signature showing the direct and indirect environmental flows supporting the city of Rome in the year 2002 (expressed in emergy units, seJ/year).

increase of source complexity, likely attributable to the increased technological development.

An opposite trend is shown by the time series of emergy data calculated for the Italian agricultural system as a whole and the agricultural sector of the Campania Region (Southern Italy) (Zucaro et al., 2010; Zucaro, 2010). Results (Table 3a and b) suggest a sharp decrease of the $\Delta S D(\%)$ for both systems: the national agriculture drops from $75.6 \%$ to $62.4 \%$, while the regional system decreases from $73.9 \%$ to $63.4 \%$.

Finally, the application of Eqs. (7) and (8) to the emergy evaluation of the economies of Nicaragua (Cuadra and Rydberg, 2000), Latvia (Ulgiati and Cialani, 2005), Denmark (Ulgiati and Cialani, 2005) and Italy (Cialani et al., 2005; Pereira et al., 2010; Zucaro, 2010) yields the results listed in Table $4 \mathrm{a}$ and b. Results show a source diversity $S D$ around 0.75 for all the investigated countries except for Italy, where it is around 0.99 . These values translate into a $\triangle S D(\%)$ in the range $66-70 \%$ for all countries (Table $4 a$ ), while it is $84 \%$ for Italy, suggesting for the latter a larger emergy evenness. The Italian $\triangle S D(\%)$ trend between 1989 and 2008 is provided in Table 4b.

\section{Discussion}

Systems can be analyzed and discussed under very different points of view. For example, if the focus is placed on efficiency the alternative renewable-nonrenewable is not explicitly addressed, because a system can be efficient either in using renewable emergy flows and in using nonrenewable emergy flows. Same if

Table 3

Trend of quality-adjusted source diversity indices of the agricultural system of Campania region (southern Italy) and the national agricultural system of Italy (years 1985-2006).

\begin{tabular}{lrrrr}
\hline $\begin{array}{l}\text { Regional agriculture } \\
\text { (Campania, southern Italy) }\end{array}$ & 1985 & 1993 & 2002 & 2006 \\
\hline $\begin{array}{l}\text { (a) } \\
S D\end{array}$ & 0.93 & 0.90 & 0.82 & 0.80 \\
$\quad S D_{\max }$ & 1.26 & 1.26 & 1.26 & 1.26 \\
$\quad S D / S D_{\max }(\%)$ & $73.9 \%$ & $72.1 \%$ & $65.3 \%$ & $63.4 \%$ \\
& & & & \\
National agriculture (Italy) & 1985 & 1993 & 2002 & 2006 \\
\hline (b) & & & & \\
$S D$ & 0.95 & 0.91 & 0.81 & 0.78 \\
$S D_{\max }$ & 1.26 & 1.26 & 1.26 & 1.26 \\
$S D / S D_{\max }(\%)$ & $75.6 \%$ & $72.6 \%$ & $64.8 \%$ & $62.3 \%$ \\
\hline
\end{tabular}

Table 4

Quality-adjusted source diversity indices of selected national economies (a) and trend of Italian source diversity index from 1989 to 2008 (b).

\begin{tabular}{|c|c|c|c|c|c|c|}
\hline & \multicolumn{2}{|c|}{ Nicaragua 2000} & Latvia 2002 & \multicolumn{2}{|c|}{ Denmark 2002} & Italy 2002 \\
\hline \multicolumn{7}{|l|}{ (a) } \\
\hline$S D$ & 0.75 & & 0.78 & 0.75 & & 0.99 \\
\hline$S D_{\max }$ & 1.08 & & 1.18 & 1.11 & & 1.18 \\
\hline \multirow[t]{3}{*}{$S D / S D_{\max }(\%)$} & $69.4 \%$ & & $66.1 \%$ & $67.6 \%$ & & $84.2 \%$ \\
\hline & Italy & Italy & Italy & Italy & Italy & Italy \\
\hline & 1989 & 1991 & 1995 & 2000 & 2002 & 2008 \\
\hline \multicolumn{7}{|l|}{ (b) } \\
\hline$S D$ & 0.94 & 0.92 & 0.89 & 0.93 & 0.99 & 0.94 \\
\hline$S D_{\max }$ & 1.18 & 1.18 & 1.18 & 1.18 & 1.18 & 1.18 \\
\hline$S D / S D_{\max }(\%)$ & $80.0 \%$ & $78.4 \%$ & $76.1 \%$ & $78.8 \%$ & $84.2 \%$ & $80.3 \%$ \\
\hline
\end{tabular}

focus is placed on reliance on imports: when the alternative is local-imported, the nonrenewability of resources is not addressed. Focusing on sustainability requires instead that many different aspects are taken into proper account, because sustainability is a complex concept, made up with economic, social, and environmental issues.

As already pointed out, the complexity of a system dynamics cannot only based on the raw amounts of mass and energy input flows. One joule of gasoline requires a much larger environmental support (in terms of generation process and embodied time) than one joule of wood. The input flows must be weighted in terms of the environmental support needed (higher support demand, lower probability that a given item is available): characterizing the mass and energy flows by means of environmental support factors, as in the proposed indicator, allows the calculation of a source diversity index capable to account for both quantity and quality of each flow.

The time series of calculated $\Delta S D(\%)$ shed light on a systems evolution towards higher or lower complexity and resiliency (e.g., increased dependency on one kind of fuel only, or increased diet simplification):

The urban system of Rome (Table 2) shows an increase of technological complexity over time, which is expected to continue until cheap fossil energy is available.

The agricultural systems of Italy and the Campania region (Table $3 \mathrm{a}$ and b) show a declining $\Delta S D(\%)$ trend, in so suggesting increased reliance on a smaller number of driving inputs, although used in large quantities.

As far as the investigated national economies are concerned, the diversity ratio $\triangle S D(\%)$ is relatively high for Italy (where technology plays an important role) and low for Latvia (where the environmental component is still dominant) (Table 4a). Table $4 \mathrm{~b}$ shows an oscillating trend of the Italian $\triangle S D(\%)$ over time, around a value of $80 \%$. Results suggest that technology and globalized markets provided Italy with a high ability to diversify its supporting sources. However, this ability was not constant, depending on economic dynamics, partially affected by fluctuations in purchasing power over the pathway towards the new European currency (ECU and Euro) in the years 1999-2002 and before.

It may appear surprising that systems that rely more on technological development (such as urban systems and industrialized economies) may show higher source diversity indices than agricultural systems and less industrialized countries. Such a behavior seems to counter the common belief that assigns higher sustainability to less technological systems. It is important to note that the diversity ratio $\triangle S D(\%)$ is not suggested here as an indicator of reliance on renewables or nonrenewables, nor it is suggested as an indicator of sustainability. A high source diversity ratio only means that the system relies on a larger set of resource options that make it more likely to develop complex structures (both environmental 
and human-dominated) and therefore more resilient in the face of fluctuations. The extreme negative case would be a system driven by only one category of input flows, for which the $S D$ would be equal to zero and the $S D_{\max }$ cannot even be defined. Such a system would be very endangered, because of its reliance on one resource option only (like those species that rely on very simplified diets). However, a high source diversity ratio in technological systems, although providing a comparative advantage, cannot last forever, and is therefore only a warranty of temporary higher resilience thanks to the higher complexity achieved. This is why societies should plan ahead in order to be ready to replace the sources that become no longer available or to quickly shift to a different internal organization that better fits the new source options that become available.

Further information for all systems investigated could be obtained by carefully looking at the data used for each year calculation (as in Table 1 ) in order to ascertain which items were responsible for better or worse performances. A step ahead for better assessment could be the standardization of the calculation procedure, by identifying large categories of driving sources (e.g., renewables, slowly renewables, food, minerals, fuels, energy carriers, chemicals, metals, labor and services) characterized by suitable transformity ranges for all systems, in order to keep the reference $S D_{\max }$ constant.

\section{Conclusions}

Brown et al. (2006) pointed out that a system's complexity cannot be understood if emergy flow evenness through system's hierarchical levels is not properly accounted for. Their qualityadjusted Shannon biodiversity indicator is a major step ahead in ecological studies. In the present paper we added another point of view to the emergy-based assessment of a system's complexity, by focusing on the diversity of emergy inflows as an important driver of structural and functional diversity of a system.

Both biological and technological diversities can make a system capable to react to resource availability fluctuations (resiliency), at least over a limited time interval. The source diversity indicator developed in this paper, was tested in a selected number of national economies, agricultural and urban systems over time. The indicator provides a measure of "source diversity", i.e., a measure of the system's reliance on a suitable variety of supporting emergy flows. Under the "source diversity" point of view, the emergy evenness through hierarchical levels pointed out by Brown et al. (2006) translates into the requirement for an optimum mix of high and low quality input flows for maximum power output.

\section{Acknowledgements}

Two of the Authors, S. Ulgiati and A. Zucaro, acknowledge the financial support received from the European Commission within the 7th Framework Programme, Project no. 217213, SMILE - Synergies in Multi-scale Inter-Linkages of Eco-social systems. Socioeconomic Sciences and Humanities (SSH) Collaborative Project FP7-SSH-2007-1.

\section{References}

Ascione, M., Campanella, L., Cherubini, F., Ulgiati, S., 2009. Environmental driving forces of urban growth and development. An emergy-based assessment of the city of Rome, Italy. Landscape and Urban Planning 93 (2009), 238-249.

Brown, M.T., Cohen, M.J., Bardi, E., Ingwersen, W.W., 2006. Species diversity in the Florida Everglades USA: a systems approach to calculating biodiversity. Aquatic Sciences 68 (3), 254-277.

Brown, M.T., Cohen, M.J., 2007. Emergy and network analysis. In: Fath, B.D., Jorgensen, S.E. (Eds.), Encyclopedia of Ecology. Elsevier, New York, p. 18.

Cialani, C., Russi, D., Ulgiati, S., 2005. Investigating a 20-year national economic dynamics by means of emergy-based indicators. In: Brown, M.T., Campbell, D. Comar, V., Huang, S.L., Rydberg, T., Tilley, D.R., et al. (Eds.), Theory and applications of the emergy methodology, vol. 3. The Center for Environmental Policy, University of Florida, Gainesville, FL.

Cuadra, M., Rydberg, T., 2000. Emergy evaluation of the environment and economy of Nicaragua. In: Brown, M.T. (Ed.), Emergy Synthesis. Theory and Applications of the Emergy Methodology. The Center for Environmental Policy, University of Florida, pp. 289-301.

Lloyd, S., Pagels, H., 1988. Complexity as thermodynamic depth. Annals of Physics $188,186-213$.

Margalef, R., 1968. Perspectives in Ecological Theory. The University of Chicago Press, Chicago, $111 \mathrm{pp}$.

Odum, H.T., 1996. Environmental Accounting: Emergy and Environmental Decision Making. John Wiley \& Sons, NY.

Pereira, L., Zucaro, A., Ortega, E., Ulgiati, S., 2010. Wealth, Trade and Environment in Brazil and Italy. An emergy-based comparison of carrying capacity, economic performance and wellbeing. In: Proceedings of Advances in Energy Studies Barcelona , 19-22 October 2010. Universitat Autonoma de Barcelona (UAB).

Ulgiati, S., Brown, M., 2006. Exploring the complexity of landscape and ecosystems. How the emergy method can be used in order to assess the flows of resources driving human activities and related impacts on ecosystem integrity. Ecological Questions 7, 85-102.

Ulgiati, S., Brown, M., 2009. Emergy and ecosystems complexity. Communications in Nonlinear Science and Numerical Simulation 14, 310-321.

Ulgiati, S., Cialani, C., 2005. Environmental and thermodynamic indicators in support of fair and sustainable policy making. Investigating equitable trade among Latvia, Denmark and Italy. In: Walter Leal Filhos, Arnold Ubelis (Eds.), Baltic Sea Region Sharing Knowledge Internally, Across Europe and Worldwide. Series on Environmental Education, Communication and Sustainability, vol. 23. Peter Lang Publisher, Frankfurt am Main, Germany, pp. 101-124.

Zucaro, A., Viglia, S., Mellino, S., Ulgiati, S., 2010. Emergy evaluation of Italian agriculture across space and time scales. In: Proceedings of Sixth Biennial Emergy Research Conference. Center for Environmental Policy , University of Florida, Gainesville, FL, 14-16 January 2010.

Zucaro, A., 2010. "Assessing the performance of agricultural systems across time and spatial scales. An extended LCA approach". A dissertation presented to the Graduate School of the University of Napoli "Parthenope" in partial fulfillment of the requirements for the requirements for the degree of Doctor of Philosophy in "Environment, Resources and Sustainable Development". 